s'est adressé à elle pour la création à Oran d'une maison de convalescence pour les légionnaires. Un appel sera adressé en vue de la réalisation de ce projet à tous les comités de province.

On voit que les associations françaises rivalisent toutes de zèle pour accomplir en faveur des soldats blessés l'œuvre généreuse et humanitaire de la Croix-Rouge.

\title{
INFIRMIÈRES MILITAIRES
}

Depuis le départ des religieuses, le soldat malade est privé des soins délicats que seule une femme peut donner. Le Ministre de la guerre français a décidé de suppléer à cette lacune par la création des infirmières militaires. C'est lì une innovation très heureuse, car, outre l'insuffisance notoire des hommes pour cetle fonction éminemment féminine, la réduction des effectifs en infirmiers exercés, dans les sections, rendait les soins nettement insuffisants. Il faut constamment faire appel aux réservistes, aux territoriaux, aux volontaires des corps de troupes pour suppléer à l'insuffisance du nombre des infirmiers.

M. le médecin-inspecteur Delorme, directeur de l'hôpital militaire d'instruction du Val de Grâce, dans un article du Caducée ${ }^{1}$ salue avec joie la création des infirmières militaires, mais en déclarant d'emblée qu'elles ne pourront donner, en service actif, le concours qu'on est en droit d'attendre de leur dévouement que si elles sont exclusivement placées sous la direction et la dépendance du médecin traitant. \&ui seul, drt-il, doit avoir action directe sur elles, sous le contrôle, bien entendu, du médecin-chef, auquel seraient soumises les plaintes que les officiers d'administration pourraient avoir à formuler contre les infirmières à l'occasion du service général. Cela est capital, car si ces infirmières dépendaient tout à fois du médecin traitant et de l'officier d'administration, ou d'autres personnes, eiles seraient prises entre le marteau et l'enclume et obligées d'employer toute leur intelligence et le meilleur de leur temps à éviter de mécontenter

$11908, \mathrm{n}^{\circ} 2$, p. 24. 
médecins et comptables dont les vues hygiéniques ou autres sont si souvent dissemblables pour ne pas dire opposées. A cette seule condition, les infirmières militaires pourront rendre les services qu'on attend de leur dévouement

Dans un article subséquent du Caducée ${ }^{1}$, le rédacteur de ce journal revenant sur la question des infirmières militaires constate que la presse médicale sans réserve a applaudi à cette innovation. Là où il y a des réserves, c'est en ce qui regarde son application. La Garde-malade hospitalière, organe des écoles de gardes-malades système Florence Nightingale, reproche en particulier au projet l'omission d'une direction morale indispensable pour un groupement de jounes filles de vingt-et-un à vingt-cinq ans. Cette direction morale, dit ce journal, ne peut être exercée que par une femme plus âgée, ayant elle-même fait des études complètes et exercé dans un hòpital les fonctions de garde-malade et de directrice. Le Caducée approuve entièrement cette réserve de l'organe des gardes-malades ; il appuie de même sur l'importance qu'il y a à donner la préséance, dans le choix des gardes, à celles qui, âgées de plus de vingt-cinq ans, ont acquis, par une pratique prolongée dans les hôpitaux mixtes, une compétence que ne sauraient avoir les plus jeunes. Le concours auquel on comple soumettre les gardes n'équivaut pas aux services rendus dans la pratique.

Dans la Presse médicale ${ }^{2}$, le $\mathrm{D}^{\mathrm{r}}$ Letulle, dont on connaît la compétence dans ces questions, dit avec raison à ce sujet :

a L'armée prétend-elle former par ses infirmières militaires, comme il y a lieu de l'espérer, un corps professionnel de choix, soigneuseınent sélecté dès la première heure et dans lequel, soucieuse par dessus tout de la santé de ses malades et du bon renom de ses hôpitaux, elle ne laissera entrer que des collaboratrices de itout repos? S’il en est ainsi, si vraiment le corps de santé militaire accepte sans arrière-pensées celte remarquable révolution sociale, qu'a-t-il besoin d'un concours? Que ne recrute-t-il pas, une à une, après une enquête minutieuse et individuelle, chacuıe de ses gardes-malades, de ses sous-surveillantes et surveillantes, voire même, s'il y a lieu, ses directrices d'hôpital; les aléas d'un examen oral et les votes d'un jury, si intègre, si émi-

$1 \mathrm{~N}^{\circ} 4$, p. 53.

215 janvier 1908 . 
nent soit-il, sont-ils comparables au choix méthodique et sagace d'une commission de classement? )

" Nommer au concours des infirmières, même militaires, apparait aux yeux les moins prévenus comme un danger, comme une source d'insuccès pour une institution aussi utile que désirable; car celte formule surannée de sélection risque de fausser, dans son mécanisme même, l'œuvre nouvelle si sagement entreprise et múrie par le Ministre de la guerre."

Sans vouloir émettre un avis quelconque dans un débal qui ne nous concerne pas, nous croyons savoir que l'opinion émise ici par le $D^{r}$ Letulle, s'est trouvée confirmée ailleurs par des expériences vécues; du reste, pour tout ce qui concerne une activité professionnelle, qui doit nécessairement être beaucoup plus prati. que que théorique, qui doit être une expérience plus qu'une science, l'épreuve du stage pratique prolongé vaudra toujours mieux que le plus brillant concours; celui-ci pourrait du reste plutôt être réservé pour l'admission à celui-là.

Quoi qu'il en soit, nous saluons avec joie la rentrée des femmes comme infirmières militaires dans l'armée où les gardes-malades des sociétés de secours de la Croix-Rouge les ont déjả précédées dès longtemps à la satisfaction de tous.

Dr Ferrière.

\section{CHIENS SANITAIRES ET CHIENS DE TRANSPORT}

Un concours international de dressage de chiens sanitaires a réuni, en janvier dernier, à Paris, devant une assistance nombreuse et en présence de délégués du Ministère de la guerre et de la Société de secours aux blessés militaires, un cerlain nombre de chiens dressés pour la recherche des blessés. Le résultat de ce premier concours a été fort intéressant et l'on peut dès maintenant envisager comme nettement efficace le concours de cet intelligent animal sur le champ de bataille.

Ont pris part au concours des chiens des types berger, airedale et dobermann, dressés en Allemagne, en Suisse et en France. Le 